\section{Cross-sectional study on differences in pain perception and behavioral distress during venipuncture between Italian and Chinese children}

\author{
Sofia Bisogni,1,2 Marta Calzolai,2 \\ Nicole Olivini,2 Daniele Ciofi, 2,3 \\ Nicola Mazzoni, 2 Simona Caprilli, 3 \\ José Rafael Gonzalez Lopez, ${ }^{4}$ \\ Filippo Festini2,3
}

1School of Nursing, University of L'Aquila, Italy; ${ }^{2}$ Department of Health Sciences, University of Florence, Italy; 3Nursing Research Unit Meyer Children Hospital of Florence; 4Faculty of Nursing, University of Sevilla, Spain

\section{Abstract}

Venipuncture is perhaps the scariest aspect of hospitalization for children as it causes pain and high levels of behavioral distress. Pain is a complex experience which is also influenced by social factors such as cultural attitudes, beliefs and traditions. Studies focusing on ethnic/cultural differences in pain perception and behavioral distress show controversial results, in particular with regards to children. The aim of this paper is to evaluate differences in pain perception and behavioral manifestations between Italian and Chinese children undergoing a venipuncture, through a cross-sectional study. Behavioral distress and self-reported pain were measured in Chinese and Italian outpatient children during a standardized blood-drawing procedure, using the Observational Scale of Behavioral Distress (OSBD) and pain scales. We observed 332 children: 93 Chinese and 239 Italian. Chinese children scored higher than Italians on pain scales - mean scores 5.3 (95\%CI 4.78-5.81) vs. 3.2 (95\%CI 2.86-3.53) - but lower mean OSBD scores - mean 4.1 (95\%CI 3.04-5.15) vs. 8.1 (95\%CI 7.06-9.14). Our data suggest that Chinese children experience higher levels of pain than their Italian peers, although they show more self-control in their behavioral reaction to pain when experiencing venipuncture.

\section{Introduction}

Preventing the perception of pain in sick children is among the primary duties of each Healthcare Professional (HCP). ${ }^{1}$ Inadequacy in evaluating the perception of pain leads to misjudge and undertreat a child's suffering; it also largely contributes to enhance the sickness-related stress experienced by a patient and by its family during hospitalization. ${ }^{1}$

It is well proven that pain is a complex phenomenon involving biological, psychological and social variables, such as race and ethnicity. ${ }^{2-4}$ With respect to adults, there is extensive literature on the role played by ethnicity and race in experiencing pain. ${ }^{5-10}$ Despite some inconsistencies, the literature suggests that ethnic differences in pain perception exist. For example, some studies suggest that Italians exhibit pain more than Old Americans do and that African Americans have lower pain thresholds to cold, heat and pressure.5,6

Differences in pain perception between different ethnic groups are likely to be influenced by genetic and biological factors. ${ }^{11,12}$ For example, specific differences related to the metabolism of analgesic drugs have been reported between people belonging to different sociocultural groups: the metabolism of codeine into its active form (morphine) depends on an enzyme pathway that can be deficient or inactive in 9\% of Europeans, 1\% of Arabs and 30\% of Hong Kong Chinese. ${ }^{11}$ Different sensitivity to pain when comparing African-American to Caucasian women has been related to diverse oxytocin levels in the two groups. 12

Culture can also exercise a powerful influence in pain perception and its manifestations: a Thai social rule called Kreng jai (literally awe heart or deferential heart) has been reported to condition Thai children in showing pain. ${ }^{13}$ Culturally constrained behavior, such as Kreng jai, may interfere with a child's oral and behavioral expression of pain, although not necessarily with pain perception. This, in turn, interferes with the health providers' awareness of pain. 14 With respect to children, it is well accepted that ethnicity plays an important role principally in evaluating pain, even though further studies are needed in this field. ${ }^{15,16}$ Some studies have already analyzed differences between Anglo-American, Caucasian children and Hispanic children, 16,17 African-American children and children of both groups. ${ }^{18,19}$

Understanding the role played by ethnicity and culture in the experience of pain is vital in order to effectively assess and manage pain, particularly in countries with recent and significant migratory flows. Pain expressed by minorities is indeed more likely to be underestimated. ${ }^{20}$

In the last 25 years, Italy has experienced a large influx of immigrants from the People' Republic of China and the number of Chinese children living in Italy - considering both immigrants and those who were born in our country - has been increasing. ${ }^{21}$ In some areas, such as Tuscany, Chinese children rep-
Correspondence: Filippo Festini, University of Florence, Department of Health Sciences, Viale Pieraccini 24, 50139 Florence, Italy.

Tel.: +39.347.210.8993 - Fax: +39.055 .566 .2577 .

E-mail: filippo.festini@unifi.it

Key words: pain, venipuncture, Chinese, distress, fear.

Contributions: SB and FF conceived and coordinated the study; SB, FF, MC and SC participated in the design of the study; SB, MC, DC, NO and NM collected data, NO, DC and NM processed data; JRGL performed the statistical analysis.

Conflict of interests: the authors declare no potential conflict of interests.

Funding: the study was entirely funded by the Department of Health Science of the University of Florence.

Conference presentation: the results of this study were presented at the 4th Congress of the European Academy of Pediatric Societies (EAPS). Istanbul, Turkey, 5-9 October 2012

Received for publication: 6 October 2014.

Accepted for publication: 5 November 2014

This work is licensed under a Creative Commons Attribution NonCommercial 3.0 License (CC BYNC 3.0).

(C) Copyright S. Bisogni, et al., 2014

Licensee PAGEPress, Italy

Pediatric Reports 2014; 6:5660

doi:10.4081/pr.2014.5660

resent a large portion of those who access the healthcare services provided by our children's hospitals. ${ }^{22}$ Therefore, it is essential to assess and manage Chinese children's pain in the best possible way. This is particularly important if we consider that only a very small number of Chinese people living in Italy work as a healthcare professional in the Italian National Healthcare System. ${ }^{23}$

According to Finley, ${ }^{14}$ to consider Chinese children more stoic than Caucasian ones is an untrustworthy prejudice. It is treacherous to expect certain social or behavioral constrictions from individuals who seem to have a certain cultural heritage. Believing that a child with an East Asian appearance comes from a Chinese heritage and that for this reason he/she is expected to show stoicism is cultural stereotyping - not sensitivity - and it will lead to misapprehend the patient's pain severity. ${ }^{14}$ Although some specific characteristics of Chinese children's pain perception have been described, ${ }^{24}$ there are scanty data regarding differences in the expression of pain between Chinese children and other ethnic groups. A Canadian study suggests that differences in 
the response to pain in relation to culture may exist as early as 2 months of age and that infants of Asian background exhibit a greater response to pain than babies of non-Asian background. ${ }^{24}$ On the other hand, another study shows that Chinese little girls have less marked facial expressions than European children. ${ }^{25}$ This aspect can interfere with the measurement of pain perception through the most commonly used assessment tools. As suggested by literature review, further studies are needed to clarify whether there is a difference in the way Chinese children perceive and display pain, in order to capably recognize and prevent their suffering, even in a socio-cultural setting that is different from their native environment.

\section{Materials and Methods}

\section{Purpose}

The primary objective of this study is to assess the possible differences existing between Italian and Chinese children - considering both those who were born on Italian soil and immigrants - with regard to the way they perceive and express pain when undergoing a standardized minor invasive procedure. Through data collection, we will be able to gain knowledge that could be helpful in improving the cognitive-behavioral and relation-based approaches that are currently used to reduce children's anxiety, pain and distress when coping with an invasive procedure. Acquiring new knowledge in this field is important in order to properly assess and identify pain in children whose expressions are not consistent with the cultural standards of a health professional, thus exposing children to the possibility of their suffering being underestimated and undertreated.

\section{Methods}

We carried out a cross-sectional analytical study which included children aged 3 to 14 years. The inclusion criteria were the following: having both Chinese parents (either born on Italian soil or immigrants) or both Italian parents; not suffering from any chronic disease according to the definition of the National Center for Health Statistics; 26 undergoing venipuncture for the first time; whose parents did not want to use any pharmacological or non-pharmacological method for pain reduction.

The study participants were selected among children attending the outpatient blood draw services (BDS) of two Italian Children's Hospitals (the Meyer Children's Hospital of Florence and the Misericordia e Dolce Hospital of Prato). We used accidental, non-probability, convenience sampling. The participation to the study was proposed to the parents of all the children who accessed the BDS consecutively during a period of two months. All children underwent a standardized venipuncture procedure, with the same needle type and gauge (23G) being used. At least one of the parents participated to the procedure. The venipunctures were performed by five very experienced nurses, none of whom was Chinese. For every child we recorded their ethnic group, age and gender. The children were observed for distress behaviors and pain during the procedure.

Distress behaviors are defined as those verbal and non-verbal actions shown by a child during an invasive procedure that produce some amount of discomfort. 27

In order to measure distress behaviors, we used the Observation Scale of Behavioral Distress (OSBD) described by Eliott et al. ${ }^{28} \mathrm{~A}$ trained observer recorded the presence or absence of eight operationally defined behaviors which indicate discomfort (cry, scream, physical restraint, verbal resistance, emotional support seeking, information seeking, verbal pain and flail) at 15 second intervals throughout the procedure, giving them a weight according to the severity of distress shown by the child. The weighted scores were summed for each 15 second interval and then divided by the number of intervals to obtain a mean score. ${ }^{28}$ We chose the OSBD because the strengths of its psychometric properties have been widely established.29-31 Since an Italian version of OSBD is not available, we decided to use the original English version. To overcome possible linguistic and cultural biases, as well as to avoid possible biases related to interrater variability of scores, all the observations were performed by a single bilingual EnglishItalian mother-tongue researcher who received an adequate previous training.

Pain was self-assessed by every child using a 0-10 Wong faces rating scale for children up to 7 years of age (32) and a 0 -10 numerical rating scale for older children. The pain scores given by children were collected and recorded by the same single researcher as above.

The OSBD and pain scores of respectively Italian and Chinese children were then compared both as total populations and stratified by age ( 3 to 5 year old pre-school children, 6 to 11 year old primary school children, 12 to 14 year old secondary school children) and by gender. For each group of children, means and 95\% confidence intervals of means were calculated respectively for OSBD scores and pain scores. Samples' size power was also calculated. The statystical softwares Epilnfo ver. 7.1.2.0 2013 and WinPEPI ver.11.43 2014 were used.

\section{Results}

Overall, we observed 332 children undergoing venipuncture: 93 of them were Chinese and 239 Italian. None of the parents of the children who accessed the BDS consecutively during the period of study and who met the inclusion criteria refused their consent to participation, therefore there were no missing subjects. The mean age was 7.59 years and girls accounted for $54.2 \%$ of the study population $(n=180)$. Table 1 shows the differences between Chinese and Italian subjects according to gender and age. There were no statistically significant differences between the two groups. Table 2 shows the overall differences in pain and OSBD scores between Chinese and Italian children.

Table 1. Differences between the two groups of children undergoing venipuncture with regard to ethnicity, gender and age. Chinese children $(n=93) \quad$ Italian children $(n=239)$

Mean age, years (SD, CI 95\%) $\quad 7.35(1.8,6.97-7.72) \quad 7.61(2.62,7.27-7.94)$

Girls, \% (CI 95\%) $58.1(47.9-67.8)$ $52.7(46.4-59.0)$

Table 2. Differences in the mean pain and Observational Scale of Behavioral Distress (OSBD) scores of Chinese and Italian children (overall).

\begin{tabular}{|c|c|c|c|}
\hline & $\begin{array}{c}\text { Chinese children } \\
\qquad(n=93)\end{array}$ & $\begin{array}{l}\text { Italian children } \\
\quad(\mathrm{n}=239)\end{array}$ & Sample power \\
\hline $\begin{array}{l}\text { Mean pain score } \\
\text { (SD, CI 95\%) }\end{array}$ & $\begin{array}{c}5.3 \\
(2.51,4.78-5.81)\end{array}$ & $\begin{array}{c}3.2 \\
(2.65,2.86-3.53)\end{array}$ & $100 \%$ \\
\hline $\begin{array}{l}\text { Mean OSBD score } \\
\text { (SD, CI 95\%) }\end{array}$ & $\begin{array}{c}4.1 \\
(5.12,3.04-5.15)\end{array}$ & $\begin{array}{c}8.1 \\
(8.13,7.06-9.14)\end{array}$ & $99.27 \%$ \\
\hline
\end{tabular}




\section{Pre-school children (age 3 to 5)}

Eighty-seven preschool children were observed, 24 were Chinese and 63 Italians. The two groups did not differ with regard to mean age and sex distribution: Chinese children's mean age was 4.61 years (SD 0.86, 95\%CI 4.21$5.00)$ while Italian children's mean age was 4.51 years (SD 0.83, 95\%CI 4.30-4,71); females accounted for $58.3 \%$ (CI95\% 38.2-76.5) of Chinese children and for $65.1 \%$ (95\%CI 52.7 76.1) of Italian Children. Table 3 shows the differences in pain and OSBD scores between Chinese and Italian pre-school age children.

\section{Primary school children (age 6 to 11)}

Two-hundred and sixteen preschool children were observed, of which 68 were Chinese and 148 Italians. In this case too the two groups did not differ with regard to mean age and sex distribution: Chinese children's mean age was 7.76 years (SD 1.20, 95\%CI 7.46-8.05) while Italian children's mean age was 7.96 years (SD $1.43,95 \%$ CI 7.72-8.19); females accounted for 57.4\% (CI95\% 45.4-68.7) of Chinese children and for $53.4 \%$ (95\%CI 45.3-61.3) of Italian Children. Table 4 shows the differences in pain and OSBD scores between Chinese and Italian primary school age children. With regard to secondary-school (age 12-14 year) children $(\mathrm{n}=29)$, no statistically significant differences in the pain and OSBD scores were found.

\section{Gender differences}

Table 5 shows differences in the mean pain and OSBD scores between Chinese and Italians stratified by gender. Within each gender group, the differences between Chinese and Italian children reflect those of the whole population: in both female and male Chinese children self-rated pain scores are higher and OSBD scores are lower than respectively in Italian females and males children.

\section{Discussion}

The results of our study show that the mean pain scores of Chinese children were significantly higher than the ones of Italian children, whereas the OSBD scores were lower. This finding is confirmed also when stratifying data for age and gender, although in one case statistical significance and adequate power were not reached, probably due to the small size of the sample. For our study, we followed a widespread accepted definition of pain: an unpleasant sensory and emotional experience associated with actual or potential tissue damage, or described in terms of such damage. ${ }^{33}$ Pain is considered a subjective and highly individual experience. This is why self-report is consid- ered the gold standard of pain assessment. ${ }^{34}$ In non verbal people pain may also be measured by a different person using behavioral and physiologic signs. ${ }^{35}$ Distress behaviors are the complex of verbal and non-verbal actions shown by a person during a painful experience. ${ }^{29}$ In our study, we used both self-rating made by the child itself and a measurement of exterior manifestations of discomfort shown by the child through their behavior, with the purpose to get a more complete vision of possible differences in discomfort experiences between Chinese and Italian children during venipuncture. Indeed, as shown by previous literature, cultural differences may influence a person's exterior manifestations of pain. ${ }^{13}$

Self-rating pain is possible in verbal children since age 3 . For illiterate children visual analog scales (VAS) are widely used. ${ }^{36}$ The self-rating scale for younger verbal children most frequently used worldwide is the Wong scale, $32,36,37$ therefore we decided to use it in this study.

Previous literature has well described the existence of a difference between genders in pain perception. ${ }^{38}$ However, this factor did not influence our results, given that there were no statistically significant differences in the male/female ratio between Chinese and Italian children and on the light of the results shown in Table 5.

Pain is a multifactorial experience, which is influenced by numerous variables. ${ }^{36}$ In order to avoid as many confounding factors as possible, we intentionally decided not to include in our study children affected by chronic diseases and to limit the evaluation of pain experience differences only to a single type of acute, invasive pain. This is because in children, having a chronic disease has the potential to increase the perception of pain intensity, $36,39-41$ and acute and chronic pain have different physiopathologic patterns and effects on perceived discomfort. 36 As a consequence, any possible inference from our results can only apply to venipuncture pain and to children without a chronic condition. Our study has some limitations. Firstly, the number of participants is limited. Secondly, the subjects were not randomly chosen, which would have made our results more robust. Finally, classifying a person as Chinese may result in ignoring potentially sig-

Table 3. Differences in the mean pain and Observational Scale of Behavioral Distress (OSBD) cores of pre-school Chinese and Italian children.

\begin{tabular}{lccc} 
& $\begin{array}{c}\text { Chinese children } \\
(\mathrm{n}=93)\end{array}$ & $\begin{array}{c}\text { Italian children } \\
(\mathrm{n}=239)\end{array}$ & $\begin{array}{c}\text { Sample power } \\
\text { Mean pain score }\end{array}$ \\
$\begin{array}{l}7.06 \\
(\mathrm{SD}, \mathrm{CI} 95 \%)\end{array}$ & 4.58 & $96.18 \%$ \\
$\begin{array}{l}\text { Mean OSBD score } \\
\text { (SD, CI 95\%) }\end{array}$ & $8.26-7.85)$ & $(3.03,3.79-5.32)$ & $29.17 \%$ \\
\hline
\end{tabular}

Table 4. Differences in the mean pain and Observational Scale of Behavioral Distress (OSBD) scores of primary school Chinese and Italian children.

\begin{tabular}{lccc} 
& $\begin{array}{c}\text { Chinese children } \\
(\mathrm{n}=93)\end{array}$ & $\begin{array}{c}\text { Italian children } \\
(\mathrm{n}=239)\end{array}$ & Sample power \\
Mean pain score & 4.9 & 2.8 & $100 \%$ \\
(SD, CI 95\%) & $(2.44,4.30-5.49)$ & $(2.42,2.40-3.19)$ & \\
Mean OSBD score & 2.4 & 6.8 & $99.8 \%$ \\
(SD, CI $95 \%)$ & $(3.06,1.65-3.14)$ & $(7.17,5.63-7.96)$ & \\
\hline
\end{tabular}

Table 5. Differences in the mean pain and Observational Scale of Behavioral Distress (OSBD) scores of Chinese and Italian children stratified by gender.

\begin{tabular}{lccc} 
& Chinese children & Italian children & Sample power \\
Female Mean pain score (n) & $5.28(54)$ & $3.19(126)$ & $99.9 \%$ \\
SD (CI 95\%) & $2.53(4.59-5.97)$ & $2.52(2.74-3.63)$ & \\
Female Mean OSBD score (n) & $4.29(54)$ & $8.43(126)$ & $91.02 \%$ \\
SD (CI 95\%) & $5.52(2.78-5.79)$ & $8.42(6.94-9.91)$ & \\
\hline Male Mean pain score (n) & $5.36(39)$ & $3.21(113)$ & $98.8 \%$ \\
SD (CI 95\%) & $2.52(4.54-6.17)$ & $2.79(2.69-3.73)$ & \\
Male Mean OSBD score (n) & $3.82(39)$ & $7.74(113)$ & $83.7 \%$ \\
SD (CI 95\%) & $4.57(2.34-5.30)$ & $7.81(6.28-9.19)$ & \\
\hline
\end{tabular}


nificant cultural differences from the point of view of the person so classified. Within the contemporary geographical and political boundaries of China there are numerous ethnic and linguistic groups, some of which are quite obvious to outsiders, while others are only known by the members of those groups.

HCPs are in charge of recognizing, understanding and treating a patient's suffering and discomfort. Therefore, they have to be welltrained in managing pain when it manifests itself, especially in children. The cultural values of a HCP may be very different from the ones of a patient belonging to a different sociocultural context. Therefore, learning how to recognize any expression of suffering - whatever the ethnic background of the patient - is of paramount importance for health professionals. HCPs who are able to recognize cultural differences will better understand a patient's pain and discomfort, thus resulting in a more accurate assessment of pain and of behavioral responses. Our study suggests that the ways children express their feelings may differ according to their cultural and ethnic backgrounds, especially in the case of feelings of suffering and discomfort. Furthermore, our study suggests that there is the risk of underestimating pain and suffering in minority groups, thus emphasizing the importance of having adequate tools to assess pain and distress.

\section{Conclusions}

Our study shows significant differences between Italian and Chinese children during a standardized minor invasive procedure. The Chinese children reported higher pain scores than their Italian peers, even though they showed evidence of a firmer behavior and of more self-control during the procedure. The Italian children, although scoring lower on the pain scales, express their distress in a more explicit way. More research is needed to understand the possible physiological or cultural reasons underlying the phenomenon we observed. However, a possible explanation of the higher pain scores reported by the Chinese children could be that the scales commonly used to assess pain in Italian children may not be adequate to assess pain in Chinese children.

\section{References}

1. Verghese ST, Hannallah RS. Acute pain management in children. $J$ Pain Res 2010;3:105-23.

2. Free MM. Cross-cultural conceptions of pain and pain control. Proc (Bayl Univ Med
Cent) 2002;15:143-5.

3. Giordano J, Engebretson JC, Benedikter R. Culture, subjectivity, and the ethics of patient-centered pain care. Camb Q Healthc Ethics 2009;18:47-56.

4. Good M. Pain as human experience: an anthropological perspective. Irvine, CA: University of California Press; 1992.

5. Campbell CM, Edwards RR, Fillingim RB. Ethnic differences in responses to multiple experimental pain stimuli. Pain 2005;113:20-6.

6. Zborowski M. Cultural components in responses to pain. J Soc Issue 1952;8:1630.

7. Hastie BA, Riley JL, Fillingim RB. Ethnic differences and responses to pain in healthy young adults. Pain Med 2005;6:6171 .

8. Rahim-Williams FB, Riley JL, Herrera D, et al. Ethnic identity predicts experimental pain sensitivity in African Americans and Hispanics. Pain 2007;129:177-84.

9. Edwards RR, Moric M, Husfeldt B, et al. Ethnic similarities and differences in the chronic pain experience: a comparison of African American, Hispanic, and White patients. Pain Med 2005;6:88-98.

10. Edwards RR, Doleys DM, Fillingim RB, Lowery D. Ethnic differences in pain tolerance: clinical implications in a chronic pain population. Psychosom Med 2001;63: 316-23.

11. Williams DG, Hatch DJ, Howard RF. Codeine phosphate in paediatric medicine. Brit J Anaes 2001;86:413-21.

12. Grewen KM, Light KC, Mechlin B, Girdler SS. Ethnicity is associated with alterations in oxytocin relationships to pain sensitivity in women. Ethn Health 2008;13:219-41.

13. Forgeron PA, Jongudomkarn D, Evans J, et al. Children's pain assessment in northeastern Thailand: perspectives of health professionals. Qual Health Res 2009;19:7181.

14. Finley GA, Kristjánsdóttir 0, Forgeron PA. Cultural influences on the assessment of children's pain. Pain Res Manag 2009;14: 33-7.

15. Fortier MA, Anderson CT, Kain ZN. Ethnicity matters in the assessment and treatment of children's pain. Pediatrics 2009;124:378-80.

16. Pfefferbaum B, Adams J, Aceves J. The influence of culture on pain in Anglo and Hispanic children with cancer. J Am Acad Child Adolesc Psychiatry 1990;29:642-7.

17. Jacob E, Sambuco G, McCarthy KS, Hockenberry M. Intensity, location, and quality of pain in Spanish-speaking children with cancer. Pediatr Nurs 2008;34:4552.

18. Widmalm SE, Christiansen RL, Gunn SM. Race and gender as TMD risk factors in children. Cranio 1995;13:163-6.

19. Yen K, Kim M, Stremski ES, Gorelick MH. Effect of ethnicity and race on the use of pain medications in children with long bone fractures in the emergency department. Ann Emerg Med 2003;42:41-7.

20. Mossey JM. Defining racial and ethnic disparities in pain management. Clin Orthop Relat Res 2011;469:1859-70.

21. Festini F, Focardi S, Bisogni S, et al. Providing transcultural nursing to children and parents: an exploratory study from Italy. J Nurs Scholarsh 2009;41:220-7.

22. Festini F, Taccetti G, Repetto T, et al. Sex ratio at birth among Chinese babies born in Italy is lower than in China. J Epidemiol Community Health 2003;57:967-8.

23. ISTAT. Annuario statistico Italiano 2013. Roma: Istat; 2013.

24. Rosmus C, Johnston CC, Chan-Yip A, Yang F. Pain response in Chinese and nonChinese Canadian infants: is there a difference? Soc Sci Med 2000;51:175-84.

25. Camras LA, Bakeman R, Chen Y, et al. Culture, ethnicity, and children's facial expressions: a study of European American, Mainland Chinese, Chinese American, and adopted Chinese girls. Emotion 2006;6:103-14.

26. Collins, JG. Prevalence of selected chronic conditions: united States, 1990-1992. National Center for Health Statistics. Vital Health Stat 1997;10:78.

27. Broome ME. Relationships between cognitive behavioral techniques, temperament, observed distress, and pain reports in children and adolescents during lumbar puncture. J Ped Nurs 1998;13:48-54.

28. Elliott CH, Jay SM, Woody P. An observation scale for measuring children's distress during medical procedures. J Pediatr Psychol 1987;12:543-51.

29. Tucker CL, Slifer KJ, Dalqhuist LM. Reliability and validity of the brief behavioral distress scale: a measure of children's distress during invasive medical procedures. J Pediatr Psychol 2001;26:51323.

30. Blount RL, Loiselle KA. Behavioural assessment of pediatric pain. Pain Res Manage 2009;14:47-52.

31. Kanagasundaram SA, Lane LJ, Cavalletto BP, et al. Efficacy and safety of nitrous oxide in alleviating pain and anxiety during painful procedures. Arch Dis Child 2001;84:492-5.

32. Wong DL, Baker CM. Pain in children: comparison of assessment scales. Pediatr Nurs 1988;14:9-17.

33. Bonica JJ. The need of a taxonomy. Pain 1979;6:247-8.

34. Schiavenato M, Craig, KD. Pain assessment as a social transaction: Beyond the gold standard. Clin J Pain 2010;26:667-6. 
35. Arif-Rahu M, Fisher D, Matsuda Y. Biobehavioral measures for pain in the pediatric patient. Pain Manag Nurs 2012;13:157-60.

36. Kuttner L. A child in pain: what health professionals can do to help. Bancyfelin: Crown House Publishing; 2010.

37. Chiaretti A, Pierri F, Valentini P, et al. Current practice and recent advances in pediatric pain management. Eur Rev Med
Pharm Sci 2013;17:112-26.

38. Fowler-Kerry S, Lander J. Assessment of sex differences in children's and adolescents' self-reported pain from venipuncture. J Pediatr Psychol 1991;16:783-93.

39. Duff AJA. Incorporating psychological approaches into routine paediatric venepuncture. Arch Dis Child 2003;88:9317.

40. Muris P, Merckelbach H. The etiology of childhood specific phobia: a multifactorial model. In: Vasey MW, Dodds MR, ed. The developmental psychopathology of anxiety. New York: Oxford University Press; 2001.

41. Festini F, Neri S, Crinelli S, et al. Pain perception related to venipuncture in children with cystic fibrosis, compared to healthy children. Int Nurs Perspect 2008;8:43-8. 